\title{
Simulation of VoLTE Services for QoE Estimation
}

\author{
Alessandro Vizzarri ${ }^{1} \quad$ Fabrizio Davide ${ }^{2}$ \\ ${ }^{1}$ Department of Enterprise Engineering, University of Rome Tor Vergata, Italy, \\ alessandro.vizzarri@uniroma2. it \\ ${ }^{2}$ Department of Innovation and Information Engineering, Guglielmo Marconi University, Italy, \\ f.davide@unimarconi.it
}

\begin{abstract}
One the most important features of a Long Term Evolution (LTE) system is the high transmission data rate in downlink and in uplink. This is not sufficient for a good Quality of Experience (QoE) perceived by the end user. The Mobile Network Operator (MNO) has to adopt appropriate techniques for an effective management of the Quality of Service (QoS) not only for bandwidth-consuming applications as video streaming but also for voice application as Voice Over LTE (VoLTE). These techniques can be based on the QoE/QoS correlation especially in case of a delaysensitive application as VoLTE. This paper formulates a method for the QoE estimation starting from QoS informations available at network level. Different scenarios are simulated using OPNET software tool. Results are statistically analyzed using regression cluster analysis techniques. Mathematical functions representing relationship between QoE/QoS metrics are identified.
\end{abstract}

Keywords: LTE, QoS, QoE, VoLTE, key performance indicators, regression, cluster

\section{Introduction}

Wireless telecommunication networks have enabled broadband applications with very high throughput. With the introduction of Long Term Evolution (LTE) the download data rate can reach $100 \mathrm{Mbps}$, while the upload data rate may be up to $50 \mathrm{Mbps}(3 \mathrm{GPP}, 2008)$. The Quality of Service (QoS) have been so strongly impacted by these new important capabilities that an efficient management of the LTE network is needed in order to guarantee acceptable levels of Quality of Experience (QoE) to the end users. Together with data applications, the voice application called Voice Over LTE (VoLTE) is also to be carefully managed. This implies the importance for the Mobile Network Operator (MNO) to make a reliable QoE estimation. MNOs need to integrate LTE native QoS features with other techniques that consider the entire communication chain. One approach is to correlate the QoS measured at network level to the QoE perceived by end user. In the scientific literature several mathematical models have been defined.
This work is focused on the QoE estimation for VoLTE application on the basis of network QoS indicators, called Key Performance Indicators (KPIs). The paper presents in Sect. II an overview of the related work and the proposed approach for QoE/QOS correlation in Sect. III. In Sect. IV a simulation activity is detailed and the results are analyzed in the Sect. V. Sect. VI resumes the main conclusions.

\section{Related Works}

Reference (3GPP, 2008) introduces the end-to-end QoS reference architecture for LTE systems as standardized by ETSI together with the basic management functions. A. Vizzarri et alii in (Vizzarri, 2013) present a review of most important papers on end-to-end QoS approach in LTE networks. Horvath et alii in (Horvath, 2013) present an innovative signalling protocol named LQSIG for the resource reservation.

A first attempt for correlating quality informations of the LTE network to those of the application is made by S. Shen et alii in (Shen, 2011). They propose a performance framework based on the mapping of the Class of Service (CoS) to the QoS. Margoc et alii in (Margoc, 2013) analyze QoS in LTE systems in order to analyze the better performances for higher priority services. Policies and strategies for priority service allocation are left to operators. This is also confirmed by Medbo et alii in (Medbo,2009), where different type of data traffic over LTE network are analyzed, e.g. VoIP and HTTP web browsing/video streaming.

In (Alreshoodi,2013) QoS/QoE correlation is studied assuming QoS as a source of disturb for QoE. In (Alreshoodi,2013) fuzzy-logic approach is proposed for QoS/QoE mapping. In (Truong, 2012) the QoS/QoE mapping for IPTV service is made on the basis of the Mean Opinion Score (MOS) (intended as the QoE metric) delay, jitter and Packet Loss Rate (intended as the QoS metrics). A. Vizzarri in (Vizzarri, 2014) analyzes the impact of the voice codec on the end-to-end QoS for a VoLTE service. In [Vizzarri, 2014) A. Vizzarri studies the impact of the network congestion (in terms of link utilization) on end-to-end QoS for a VoLTE service. 


\section{Proposed Approach}

\subsection{Methodology}

LTE system enables fully IP-based applications thanks to data transmitted through Packet Switching (PS) paths. That implies VoLTE to be treated as an IP-based application (3GPP, 2008). IP Multimedia Sub System (IMS) (3GPP, 2008) and Session Initiation Protocol (SIP) are integrated with LTE network nodes (IETF, 2005).

Figure 1 shows the LTE logical architecture supporting VoLTE service includes VoLTE User Equipment (UE), Evolved Universal Terrestrial Radio Access Network (E-UTRAN), Evolved Packet Core (EPC) and IMS Core Network.

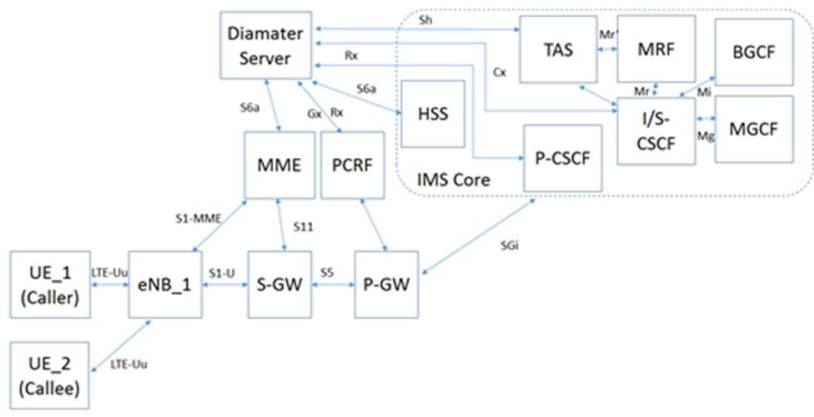

Figure 1. Logical architecture for VoLTE service [3GPP TS 23.002].

As a fully IP-based application, VoLTE is delivered over the LTE in a best effort modality. That implies the Mobile Network Operator (MNO) has to manage in order to guarantee acceptable levels of both QoS measured at the network level and QoE perceived by the end user at the application level. QoE is usually represented by a subjective measure called Mean Opinion Score (MOS). MOS is a scalar variable that measures the degree of service acceptance by the end user on range from 1 (worst case) to 5 (best case) (ITU, 2016). A MOS value equal to 5 is indicative of an Excellent Quality (imperceptible impairment), 4 of a Good Quality (perceptible but non annoying impairment), 3 of a Fair Quality (slightly annoying impairment), 2 of a Poor Quality (annoying impairment) and 1 of a Bad Quality (very annoying impairment). MOS value is derived from to $\mathrm{R}$ factor provided by ITU E-Model (ITU,2008) which takes in account several factors impacting the QoS, e.g. choose of voice codec, transmission delay, etc.

KPIs measured at the network level are typically Delay (DEL), Jitter (JIT) and Packet Loss Rate (PLR) (Yu et alii, 2007). The delay is represented as the amount of time a packet sent by source (caller) takes to reach destination (callee). Jitter is the variation in the time between packets arriving, caused by network congestion or route changes. Negative effects of Delay and Jitter are represented by phenomena of voice echoes. A high value of PLR can produce overlapping of words with a strong negative impact on voice intelligibility.

The present paper is mainly focused on the study and the identification of mathematical models for QoS/QoE correlation in different VoLTE realistic scenarios. Considered metrics are:

- QoE metrics: MOS;

- QoS KPIs: end-to-end Delay (e2eDEL), Jitter (JIT) and Packet Loss Rate (PLR).

Correlation techniques here adopted by the authors are statistical regression and cluster analysis.

\subsection{Network models}

In order to build realistic scenarios for VoLTE, network impairments are included. They are modelled on the basis of two constraints:

- Presence of mixed traffic: VoLTE application is delivered over LTE network together with HTTP web browsing application;

- Presence errors on end-to-end transmission: they are modelled inserting an IP cloud which introduces both additive delay ( 0.1 seconds) and IP packet discard ratio $(1 \%)$.

On the basis of the VoLTE logical architecture shown in Figure 1, Figure 2 reports four different LTE network models, built in order to model realistic conditions for the VoLTE application and compute QoS and QoE metrics.

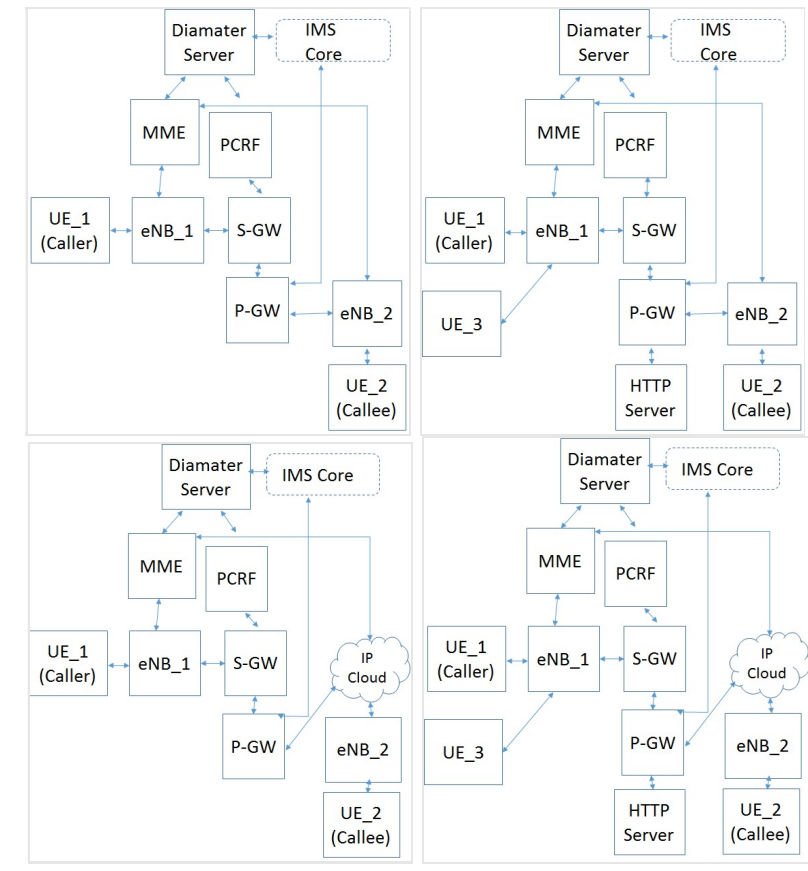

Figure 2. Four LTE network models identified to model realistic conditions for for VoLTE application. From the upper leftmost to right: UE for VoLTE without IP cloud; two UEs for VoLTE and HTTP browsing without IP cloud; One UE for VoLTE with IP cloud; two UEs for VoLTE and HTTP browsing with IP cloud. 
The first model is related to a classic VoLTE call between two LTE UEs: a caller (UE_1) and a callee (UE_2). It is the situation with a single LTE application (VoLTE) without any network impairment. The second model is related to a situation with two different services delivered over the same LTE network. VoLTE is the first application between UE_1 and UE_2; HTTP web browsing is the second one and it is performed by UE_3 contacting an HTTP server. UE 1 (VoLTE caller) and UE 3 are attached to the same eNodeB (eNB 1). The third model is an extension of the first one. VoLTE application is affected by only the insertion of an IP cloud across the end-to-end transmission chain. The fourth model is an extension of the second one. Here VoLTE application is affected by both the presence of IP cloud and the presence of a second application (HTTP web browsing) performed by another user.

\section{Simulation}

All scenarios are simulated using the LTE network model provided by OPNET 17.5 PL6.

\subsection{OPNET Settings}

Both the antenna gain equal to $-1 \mathrm{dBi}$ and the receiver sensitivity to $-200 \mathrm{dBm}$ characterize the UE. eNodeB transmission mode is FDD Duplex Mode. Link type among LTE network nodes is PPP D3: the data rate is $44.736 \mathrm{Mbps}$. Voice codec is GSM EFR with one voice frame per packet. EPS bearer has a QoS Class Identifier (QCI) equal to 1 (GBR) and an Allocation and Retention Priority (ARP) equal to 1 . Through the HTTP application, UE_3 can download $1 \mathrm{~KB}$ web page, n. 5 medium images with dimension up to $2 \mathrm{~KB}$ and two short videos with dimension up to $350 \mathrm{~KB}$. The simulation period is equal to 3 minutes for all scenarios. The simulation area is a typical campus area $\left(100 \mathrm{Km}^{2}\right)$.

\subsection{Assumptions on users and networks}

According to the four network models defined in the Sect. III, we identified 48 scenarios to be simulated.

The scenario set includes two tunable parameters:

- S1 link capacity: starting form $100 \%$ (corresponding to $1,073,741,824 \mathrm{bps}$ ), the link capacity is decreased to $75 \%, 50 \%$ and $30 \%$;

- eNodeB Bandwidth: $5 \mathrm{MHz}, 10 \mathrm{MHZ}, 20 \mathrm{MHz}$.

Table 1 reports scenarios grouped in four subsets, and their parameters.

\subsection{Results}

Table 2 gives a view of simulation results per homogeneous groups of scenarios, in terms of related QoS metrics and QoE metric. As a first remark, MOS is acceptable for the first two subsets (fair quality), and too low for the third (poor quality) and fourth (bad quality) subsets.
Table 1. Scenario Configurations.

\begin{tabular}{|c|c|c|c|c|c|c|}
\hline \multirow[b]{2}{*}{$\begin{array}{l}\text { Sub } \\
\text { set } \\
\text { No. }\end{array}$} & \multirow[b]{2}{*}{$\begin{array}{c}\text { Scen } \\
\text { ario } \\
\text { No. }\end{array}$} & \multirow{2}{*}{$\begin{array}{l}\text { LTE } \\
\text { Servi } \\
\text { ce } \\
\text { Type }\end{array}$} & \multirow{2}{*}{$\begin{array}{l}\text { S1 } \\
\text { Link } \\
\text { Capa } \\
\text { city } \\
{[\%]}\end{array}$} & \multirow{2}{*}{$\begin{array}{l}e N B \\
\text { Band } \\
{[M H z} \\
]\end{array}$} & \multicolumn{2}{|c|}{$\begin{array}{l}\text { Impairment } \\
s \\
\text { due to IP } \\
\text { Cloud }\end{array}$} \\
\hline & & & & & $\begin{array}{l}\text { Pack } \\
\text { et } \\
\text { Disc } \\
\text { ard } \\
\text { Ratio } \\
{[\%]}\end{array}$ & $\begin{array}{l}\text { Pac } \\
\text { ket } \\
\text { Lat } \\
\text { enc } \\
y \\
{[s]}\end{array}$ \\
\hline 1 & $1-12$ & VoLTE & $\begin{array}{c}100 \\
75 ; 50 \\
30\end{array}$ & $\begin{array}{c}5 ; 10 \\
20\end{array}$ & $\begin{array}{c}\text { Not } \\
\text { present }\end{array}$ & $\begin{array}{c}\text { Not } \\
\text { prese } \\
\text { nt }\end{array}$ \\
\hline 2 & $13-24$ & $\begin{array}{c}\text { VoLTE } \\
+ \\
\text { HTTP } \\
\text { Browsi } \\
\text { ng } \\
\end{array}$ & $\begin{array}{c}100 \\
75 ; 50 \\
30\end{array}$ & $\begin{array}{c}5 ; 10 \\
20\end{array}$ & $\begin{array}{c}\text { Not } \\
\text { present }\end{array}$ & $\begin{array}{c}\text { Not } \\
\text { prese } \\
\text { nt }\end{array}$ \\
\hline 3 & $25-36$ & $\begin{array}{c}\text { VoLTE } \\
+ \text { IP } \\
\text { Cloud }\end{array}$ & $\begin{array}{c}100 \\
75 ; 50 \\
30 \\
\end{array}$ & $\begin{array}{c}5 ; 10 \\
20\end{array}$ & 1 & 0.1 \\
\hline 4 & $37-48$ & $\begin{array}{c}\text { VoLTE } \\
+ \\
\text { HTTP } \\
\text { Browsi } \\
\text { ng + IP } \\
\text { Cloud } \\
\end{array}$ & $\begin{array}{c}100 \\
75 ; 50 \\
30\end{array}$ & $\begin{array}{c}5 ; 10 \\
20\end{array}$ & 1 & 0.1 \\
\hline
\end{tabular}

Table 2. Simulation Results.

\begin{tabular}{|c|c|c|c|c|c|}
\hline \multirow{2}{*}{$\begin{array}{l}\text { Sub } \\
\text { set } \\
\text { No. }\end{array}$} & \multirow{2}{*}{$\begin{array}{c}\text { Scen } \\
\text { ario } \\
\text { No. }\end{array}$} & \multicolumn{3}{|c|}{$\begin{array}{c}\text { QoS } \\
\text { Metrics }\end{array}$} & \multirow{2}{*}{$\begin{array}{l}\text { QoE } \\
\text { Metrics } \\
\text { MOS }\end{array}$} \\
\hline & & $\begin{array}{l}\text { Delay } \\
{[s]}\end{array}$ & $\begin{array}{l}\text { Jitter } \\
{[s]}\end{array}$ & $\begin{array}{l}\text { PLR } \\
{[\%]}\end{array}$ & \\
\hline 1 & $1-12$ & $\begin{array}{l}{[0.11 ;} \\
0.12]\end{array}$ & $\begin{array}{r}{[0.06 ;} \\
0.11] \\
\end{array}$ & $\begin{array}{c}{[0.21 ;} \\
0.53]\end{array}$ & $\begin{array}{l}{[3.46 ;} \\
3.70] \\
\end{array}$ \\
\hline 2 & $13-24$ & $\begin{array}{l}0.12 ; \\
0.13] \\
\end{array}$ & $\begin{array}{l}{[0.09 ;} \\
0.13]\end{array}$ & $\begin{array}{c}1.16 ; \\
1.37] \\
\end{array}$ & $\begin{array}{l}{[2.90 ;} \\
3.50] \\
\end{array}$ \\
\hline 3 & $25-36$ & $\begin{array}{c}0.19 ; \\
0.21] \\
\end{array}$ & $\begin{array}{l}0.15 ; \\
0.19]\end{array}$ & $\begin{array}{c}7.40 \\
8.85] \\
\end{array}$ & $\begin{array}{l}{[2.26 ;} \\
2.50]\end{array}$ \\
\hline 4 & $37-48$ & $\begin{array}{c}0.26 ; \\
0.27] \\
\end{array}$ & $\begin{array}{c}{[0.21 ;} \\
0.27] \\
\end{array}$ & $\begin{array}{l}{[21.60 ;} \\
26.06]\end{array}$ & $\begin{array}{l}{[1.45 ;} \\
1.70]\end{array}$ \\
\hline
\end{tabular}

\section{Statistical Analysis}

\subsection{Single Variable Non Linear Regression Model}

A generic regression model is representable as

$$
Y=f(x)+\varepsilon_{i}
$$

where $\mathrm{Y}$ is the response (dependent variable) to the predictor x (independent variable), with $\mathscr{E}_{\mathrm{i}} \sim\left(\mathrm{N}, \sigma_{\mathrm{i}}^{2}\right)$ the error for each observation. The relationship function $\mathrm{f}(\mathrm{x})$ can be linear or not. Based on available dataset the model of choice for regression is non-linear, and 
specifically exponential (Alreshoodi, 2013). In case of MOS as response and a KPI as predictor, (1) becomes

$$
Q o E=a * \exp \left(-b^{*} Q o S\right)+c
$$

where $\mathrm{a}$ and $\mathrm{b}$ are numerical coefficients. QoS may be Delay, Jitter and PLR in turn.

\subsubsection{MOS vs DEL fitting.}

In case of fitting between MOS and Delay, the regression model is

$$
\text { MOS }=-13.96 * \exp (-1.133 * D E L)-8.783
$$

Figure 3 gives a graphic representation of (3). The fitting quality is proved by a good value of R-squared $(0.9487)$ (the adjusted R-Squared is quite near, i.e. 0.9464). Further the value of Root Mean Square Error (RMSE) is low enough, i.e. 0.185 .

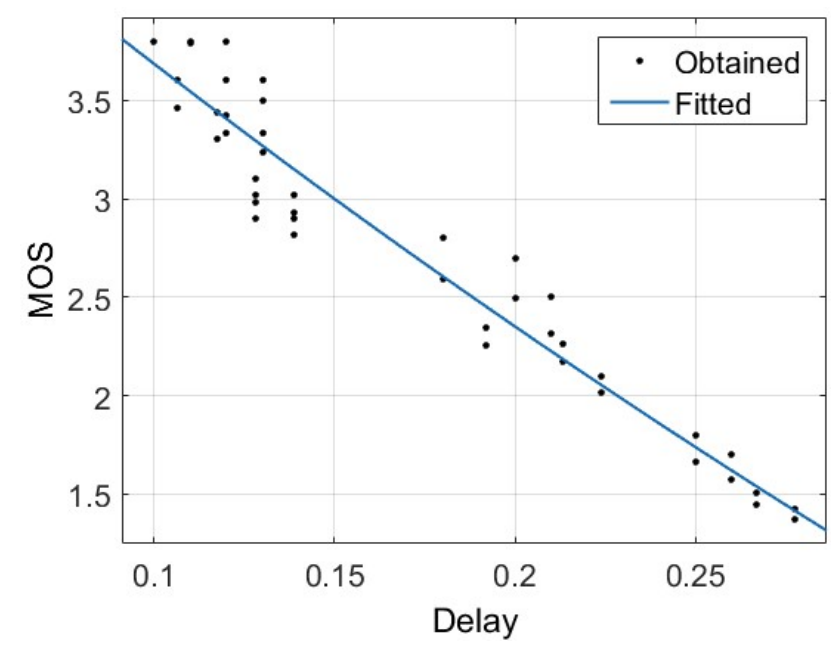

Figure 3. MOS to Delay regression model.

\subsubsection{MOS vs JIT fitting.}

In case Jitter is considered as a QoS, the regression model is estimated as

$$
M O S=-94.21 * \exp (-0.1219 * J I T)-98.78
$$

Figure 4 shows the plot for (4). R-squared value is as for (3), i.e. 0.9374 (as much as the adjusted R-squared, i.e. 0.9352 ). The RMSE value is 0.2034 . We can conclude the fit to be adequate.

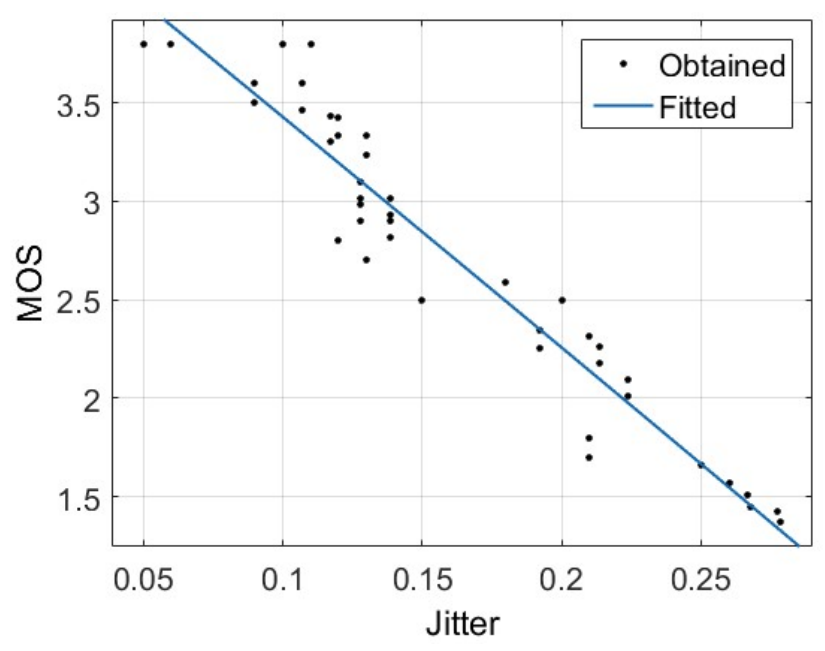

Figure 4. MOS to Jitter regression model.

\subsubsection{MOS vs PLR fitting.}

As far as PLR is considered, the regression model comes out to be

$$
M O S=2.198 * \exp (-11.06 * P L R)+1.391
$$

Figure 5 shows graphically the regression model. Data exhibit a strong non linear character. R-square value is 0.8969 , while RMSE value is 0.226 . The quality of fitting is slightly lower than for (3) and (4).

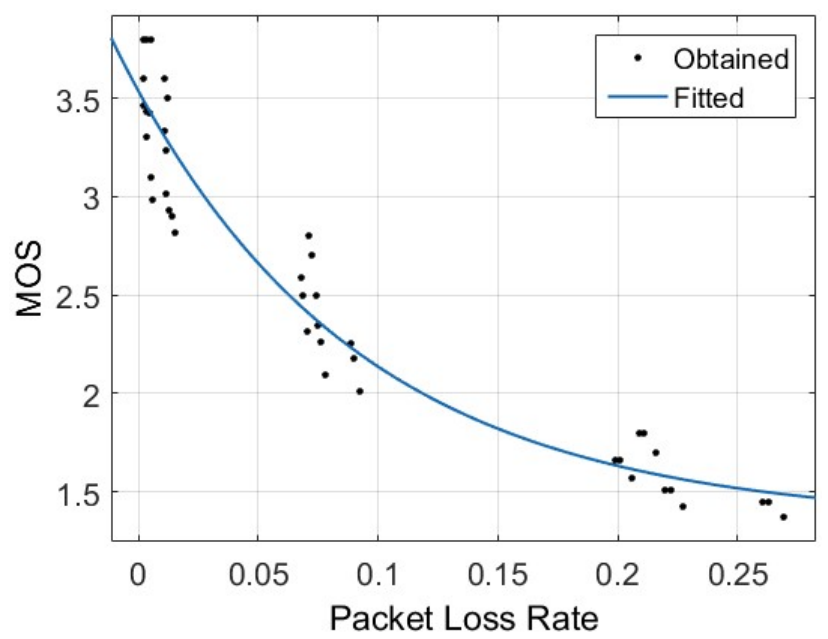

Figure 5. MOS to PLR regression model.

Further Figure 5 reports the existence of 3 clusters in the scenarios set. The clusters are linearly separable. This effect is similar in the (MOS, DEL) plane, and not evident in the (MOS, JIT) plane. 


\subsection{Multiple Variable Linear Regression Model}

The previous fitting models are able to identify a (sometimes-strong) non-linear relationship between MOS and QoS KPIs taken once a time. These models give for VoLTE an idea of the QoE/QoS mapping but have a limited validity. In this section we look for a relationship between all QoS KPIs and QoE, a multivariable approach is helpful. The Multiple variable regression (MLR) model is usually represented as

$$
Y=\beta_{0}+\beta_{1} * x_{1}+\beta_{2} * x_{2}+\ldots .+\beta_{n} * x_{n}+\varepsilon_{i}
$$

where $\beta_{0}, \beta_{1}, \beta_{2}, \ldots, \beta_{\mathrm{n}}$ are the unknown regression coefficients and $\mathscr{E}_{\mathrm{i}} \sim\left(\mathrm{N}, \sigma_{\mathrm{i}}^{2}\right)$ is the error for each observation.

As far as MOS is the response (dependent variable) and DEL, PLR, THR are the independent variables, from (6) we have

$$
M O S=\beta_{0}+\beta_{1} * D E L+\beta_{2} * J I T+\beta_{3} * P L R
$$

For our dataset, the regression model comes out as

$$
\begin{aligned}
M O S= & 4.824-6.958 * D E L-5.533 * J I T \\
& -0.1477 * P L R
\end{aligned}
$$

Figure 6 shows relevant statistical indicators for model (8): the fit looks actually good, and residuals are well distributed and adequately symmetric. The goodness of the regression model (8) is proved by Rsquared quite high 0.973 (with an adjusted R-squared equal to 0.971 ) and a quite low value of RMSE, i.e. 0.136 . The overall pValue is very low: $1.75 \mathrm{e}-34$. This means the null hypothesis can be firmly rejected.
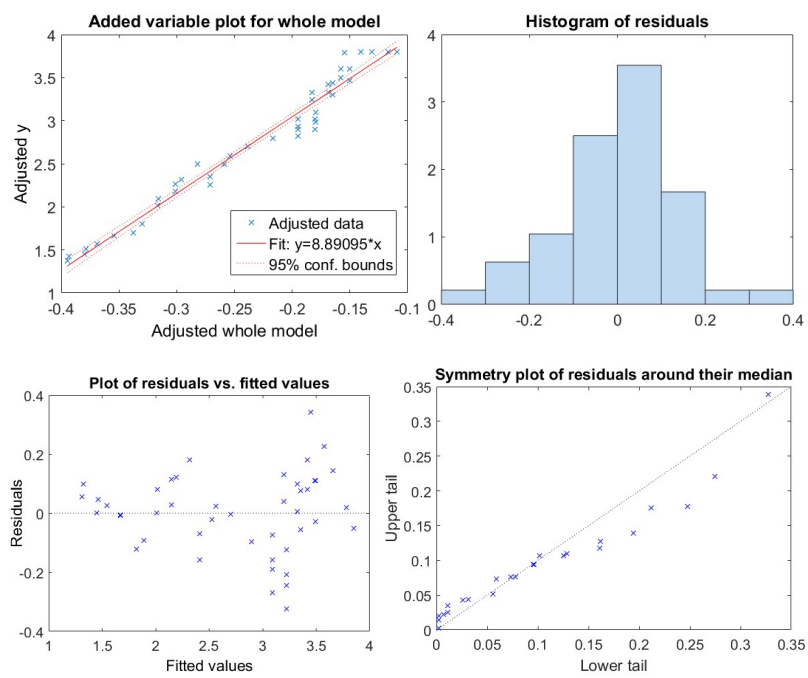

Figure 6. Statistical indicators for MLR in case of MOS, DEL, JIT and PLR. From upperleftmost to right: plot for whole model vs samples; histograms of residuals; symmetry plot of residuals around their median; residuals vs fitted values.
Table 3 presents the main statistical parameters for the coefficients in (7) and (8).

Table 3. MLR Results.

\begin{tabular}{|c|c|c|c|c|}
\hline $\begin{array}{c}\text { Coeffi } \\
\text { cients }\end{array}$ & $\begin{array}{c}\text { Estimated } \\
\text { Value }\end{array}$ & $\begin{array}{c}\text { Standard } \\
\text { Error } \\
\text { (SE) }\end{array}$ & tStat & $p$ Value \\
\hline $\begin{array}{c}\beta 0 \\
\text { (interce } \\
\mathrm{pt})\end{array}$ & 4.824 & 0.1339 & 36.035 & $2.67 \mathrm{e}-34$ \\
\hline$\beta_{1}$ & -6.958 & 1.36 & -5.116 & $6.568 \mathrm{e}-06$ \\
\hline$\beta_{2}$ & -5.533 & 0.8751 & -6.323 & $1.131 \mathrm{e}-07$ \\
\hline$\beta_{3}$ & -0.1477 & 0.6879 & -0.2147 & 0.8310 \\
\hline
\end{tabular}

\subsection{Cluster Analysis}

Besides the results coming out of the regression analysis, we need to gain a better insight in the QoE/QoS mapping. Clustering may provide that. Let us map the scenarios in the 4 dimensional feature space, whose dimensions are MOS, DEL, JIT and PLR. If we apply the well-known k-means technique [20] [21], we get three clusters as a result after the algorithm stabilization. Figure 7 shows the clusters projected onto the first two principal components. Clusters are linearly separable and fairly separated.

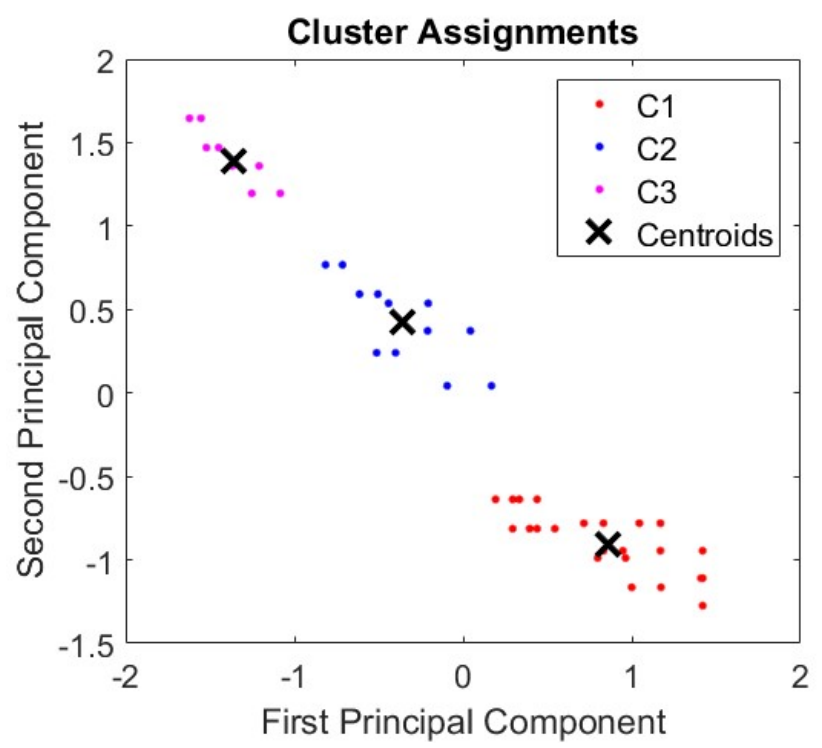

Figure 7. Clusters of VoLTE scenarios. Plot is a projection on the plane of the first two principal components. Centroids are marked as crosses.

Table 4 reports the position of the centroids of the three identified clusters in the 4-dim space. The utility of this clustering is evident from the changes of QoE between the centroids: MOS assumes the best value in $\mathrm{C} 1$ that consists of 24 scenarios, characterized by a single application: (VoLTE in the scenarios 1-12) or by 
a mixed traffic, (VoLTE and HTTP web browsing applications, scenarios 13-24).

The MOS gets worse of $-3.1 \mathrm{~dB}$ for $\mathrm{C} 2$ when the VoLTE application co-exists with end-to-end transmission errors due to an IP cloud (scenarios 25-36). The MOS decreases of $-6.8 \mathrm{~dB}$ in $\mathrm{C} 3$ where both a mixed traffic (VoLTE and HTTP web browsing) and end-to-end transmission errors are present.

Table 4. MLR Results.

\begin{tabular}{|c|l|c|c|c|c|}
\hline $\begin{array}{l}\text { Cluster } \\
\text { No. }\end{array}$ & $\begin{array}{l}\text { Scenar } \\
\text { io } \\
\text { No. }\end{array}$ & MOS & $\begin{array}{c}\text { Dela } \\
y \\
{[s]}\end{array}$ & $\begin{array}{c}\text { Jitter } \\
{[s]}\end{array}$ & $\begin{array}{c}\text { PLR } \\
{[\%]}\end{array}$ \\
\hline 1 & {$[1-24]$} & 3.40 & 0.12 & 0.11 & 0.77 \\
\hline 2 & {$[25-36]$} & 2.39 & 0.20 & 0.19 & 8.20 \\
\hline 3 & {$[37-48]$} & 1.56 & 0.26 & 0.25 & 22.54 \\
\hline
\end{tabular}

To be remarked that cluster $\mathrm{C} 1$ gathers scenarios from subsets 1 and 2. C2 is overlapping with subset 2 of the scenarios. C3 is overlapping with subset 3 . Based on Table II, Table IV shows both this relationship and related cluster characteristics.

\section{Conclusions}

VoLTE is an important service for LTE networks since it is the basic voice service in the $4 \mathrm{G}$ wireless standard. Since LTE is a fully IP-based system, VoLTE application is delivered in the best effort modality. Mapping QoE/QoS is of key importance for the MNOs. Here we studied how the correlation between QoE and QoS can be expressed through mathematical models.

We designed a certain number of scenarios, that include as network impairments both mixed traffic (copresence of VoLTE and HTTP web browsing), and endto-end transmission errors (presence of an IP cloud). We analyzed results from simulation through different techniques: single variable regression, Multi Linear Regression (MLR) and cluster analysis.

From the single variable nonlinear regression, we learnt that DEL and JIT have linear models. On the contrary, the PLR model exhibits a stronger nonlinearity, which is the reason why presence of clusters in data has been anticipated from Figure 5.

The multivariable linear regression came to results comparable with the single variable regression for dependence from DEL and JIT. This is straightforward from the linearization of (3) and (4). Further, model (8) expresses the joint influence of all QoS metrics on MOS, with a good statistical quality. Nonetheless, the coefficient related to PLR has an unsatisfying $p$ Value. This may be motivated by the irrelevance of the variable (that is the likelihood of the null hypothesis). Further, it may be a signature of statistical instability when a nonlinear behavior is forced to accommodate into a linear model. Related work demonstrated PLR to be a relevant predictor for MOS. Thus we consider (8) relevant under a theoretical viewpoint, but of limited use as far as a large PLR variation is considered.

The cluster analysis showed that the designed scenarios naturally group into three distinct clusters. Each of them has different features in terms of QoE and QoS metrics. As a first result, the authors observed that scenarios of $\mathrm{C} 3$ have an unacceptable MOS, due to errors of end-to-end transmission (modeled through IP cloud). About the role of PLR, clustering reveals a strong capability of PLR to predict existence of cluster (compare Figure 3 and Figure 7), and a very high discrimination between the clusters based on the PLR value (see Table IV)

Future works will be focused on the mapping QoE/QoS for LTE networks with different scenarios and design parameters. Applications in the scope will be video streaming and HTTP web browsing.

\section{References}

M. Alreshoodi and J. Woods. Survey on QoE/QoS correlation models for multimedia services. International Journal of Distributed and Parallel Systems, 3: 53-72, 2013.

M. Alreshoodi and J. Woods. An empirical study based on a Fuzzy Logic System to Assess the QoS/QoE Correlation for Layered Video Streaming. In Proceedings IEEE International Conference on Computational Intelligence and Virtual Environments for Measurement Systems and Applications, pages. 180-184, 2013.

G. Horvath. End-to-end QoS Management Across LTE Networks. In Proceedings 21st International Conference on Software, Telecommunications and Computer Networks, pages 1-6, 2013.

IETF RFC 4028. Session Timers in the Session Initiation Protocol. Available via https://www.ietf.org/ [accessed March 29, 2018].

ITU Recommendation P.800.2 Series P: Terminals and Subjective and objective assessment methods. Available via https://www.itu.int/ [accessed March 29, 2018].

ITU Recommendation G.109 Definition of categories of speech transmission quality. Available via https://www.itu.int/ [accessed March 29, 2018].

A. Margoc. Quality of Service in Mobile Networks. In Proceedings 55th International Symposium ELMAR, pages 263-267, 2013.

J. Medbo. Propagation channel impact on LTE positioning accuracy: A study based on real measurements of observed time difference of arrival. In Proceedings 20th International Symposium on Personal, Indoor and Mobile Radio Communications, pages 2213-2217, 2009.

J. A. Rice. Mathematical Statistics and Data Analysis. 3rd Edition, Belmont, CA, Thomson Brooks/Cole, 2009.

S.Shen. End-to-End QoS performance management across LTE networks. In Proceedings 3th Asia-Pacific Network Operations and Management Symposium, pages 1-4, 2009. 
Release 8 V0.0.3, Overview of 3GPP Release 8: Summary of all Release 8 Features. 2008. Available via www.3gpp.org/ [accessed March 29, 2018].

3GPP TS 123.207 Digital cellular telecommunications system; Universal Mobile Telecommunications System; LTE; End-to-end Quality of Service concept and architecture. Available via www.3gpp.org/ [accessed March 29, 2018].

3GPP TS 23.002 3rd Generation Partnership Project; Technical Specification Group Services and Systems Aspects; Network architecture. Available via www.3gpp.org/ [accessed March 29, 2018].

3GPP TS 29.228 Digital cellular telecommunications system; Universal Mobile Telecommunications System; LTE; IP Multimedia Subsystem $C x$ and Dx Interfaces; Signaling flows and message contents. Available via www.3gpp.org/ [accessed March 29, 2018].

3GPP TS 32.260 3rd Generation Partnership Project; Technical Specification Group Services and System Aspects; Telecommunication management; Charging management; IP Multimedia Subsystem charging. Available via www.3gpp.org/ [accessed March 29, 2018].

T.H Truong and Tai-Hung Nguyen, and Huu-Thanh Nguyen. On relationship between Quality of Experience and Quality of Service metrics for IMS-based IPTV networks. In Proceedings IEEE RIVF International Conference on Computing and Communication Technologies, Research, Innovation, and Vision for the Future, pages 1-6, 2012.

J.Urrea and N. Gaviria. Statistical performance evaluation of P2P video streaming on multi-hop wireless networks. In Proceedings 20th Symposium on Signal Processing, Images and Computer Vision, pages 1-6, 2015.

A. Vizzarri and S. Forconi. Review of Studies on End-to-End QoS in LTE Networks. In Proceedings AEIT Congress, pages 1-6, 2013.

A. Vizzarri. Analysis of VoLTE end-to-end quality of service using OPNET. In Proceedings European Symposium on Computer Modeling and Simulation, pages 452 - 457, 2014.

A. Vizzarri. Analysis of VoIP Over LTE End-To-End Performances in Congested Scenarios. In Proceedings Artificial, Intelligence, Modelling and Simulation, pages 393-343, 2014.

J. Yu and I. Al-Ajarmeh. Call admission control and traffic engineering of VoIP. In Proceedings 2nd International Conference on Digital Telecommunications, pages 11-16, 2007. 